\title{
Development of a Radio Telescope for Very Low Frequency Observations
}

\author{
Arpit Gupta ${ }^{1, *}$, Seow Kit Hint ${ }^{2}$, and Cao Shangyu ${ }^{1}$ and Hoe Teck Tan ${ }^{3}$ \\ ${ }^{1}$ Student, National Junior College, Singapore \\ ${ }^{2}$ Student, School of Science and Technology \\ ${ }^{3}$ Teacher, School of Science and Technology
}

\begin{abstract}
Sudden ionospheric disturbances are transient changes in the ionosphere caused by enhancement in X-ray and EUV fluxes during solar flare events. The Solar Storm Radio Telescope is developed to detect Very Low Frequency (VLF) signals with frequency between 3-30 kHz transmitted from various VLF stations around the Globe. We will also be investigating different methods to reduce the background noise in the data collection. This will help to ensure an accurate hit when there is a sudden ionospheric disturbance.
\end{abstract}

\section{Introduction}

A Solar Storm Radio Telescope is a device that utilises radio waves propagation and sudden ionospheric disturbance to monitor solar storms. It is used to observe and study astronomical phenomena unobservable by the human eye.

Solar storms have a very diverse effect on the earth, examples of such include sunspots influencing the weather, solar flares disrupting satellite signal transmissions to earth and the well-known coronal mass ejection (CME) which behaves very much like an electromagnetic pulse (EMP) given its ability to disrupt and wreak havoc on electronics. Although we know about solar storms and how they affect us, we don't know enough about them to take preventive action against them to safeguard human civilisation should a coronal mass ejection hit the earth. It is precisely because we know so little about solar flares that we need to study them further using a radio telescope to further deepen our understanding about solar flares and the sun, in the hopes of being able to find a solution to protect human civilisation and its technology should we ever be hit by a solar storm of devastating magnitude.

The SSRT we will be developing is designed to be low cost and easy to construct. Our radio telescope will encourage young aspiring engineers and radio enthusiasts to pick up their first project in radio astronomy due to its low cost making it affordable for anyone from students to adults alike as well as its simplicity which will allow anyone to take on this project as long as they have the fundamental technical knowledge on engineering and radio astronomy. This will also contribute to the effort of raising awareness on how important radio astronomy is to science and humanity in general.

\footnotetext{
*E-mail: $\operatorname{arpitg1011@gmail.com~}$
} 


\subsection{Hypothesis}

By building a radio telescope with an antenna composed of $12 \mathrm{AWG}$ wire and PVC pipes to pick up radio signals at very low frequencies, an amplifier circuit based around a dual 082 JFET Operational Amplifiers and a 741 type op-amp to amplify and reduce interference of the signal received, a pc with a soundcard and an audio analysing package installed on the pc to plot the data on ionospheric activity in real-time on a chart, one can observe the sun's effect on our ionosphere by studying the fluctuations in ionospheric activity.

\subsection{Engineering Goals}

To provide amateur radio enthusiasts with a low-cost and self-customisable alternative to buying radio telescopes off the shelf as well as to encourage students and adults to take up engineering if they have yet to do so and are interested through this relatively simple project involving fundamental technical knowledge on radio astronomy and electronics.

\section{Procedures}

Figures and tables, as originals of good quality and well contrasted, are to be in their final form, ready for reproduction, pasted in the appropriate place in the text. Try to ensure that the size of the text in your figures is approximately the same size as the main text (10 point). Try to ensure that lines are no thinner than 0.25 point.

\subsection{Antenna:}

1. Loop $200 \mathrm{~m}$ of 12 AWG wire around a PVC pipe structure of preferably $1.20 \mathrm{~m} \mathrm{x}$ $0.95 \mathrm{~m} \times 0.95 \mathrm{~m}$.

2. Deduce the natural resonance of the antenna by feeding a sine wave of $5 \mathrm{kHz}$ into a separate loop of the same wire. The voltage induced is measured. The frequency of the signal being fed into the single loop is gradually increased. The natural resonance of the antenna is the frequency at which the voltage peaks.

3. The antenna is then tuned by adding polypropylene capacitors in parallel to the end of the antenna, such that its natural resonance is equal to that of the VLF transmitter that will be used for VLF observation. Alternatively, changing the number of loops of wire of the antenna also changes the natural resonance of the antenna.

\subsection{Amplifier Circuit}

1. Assemble the circuit on a breadboard(excluding dotted sectors) for testing.

2. Test the circuit by feeding a sine wave from a function generator into terminal block $\mathrm{J}$, while using an oscilloscope to check for any form of signal amplification 
3. Once amplification has been proven successful, assemble the circuit on a stripboard/perf board for durability purposes and to make the circuit more compact.

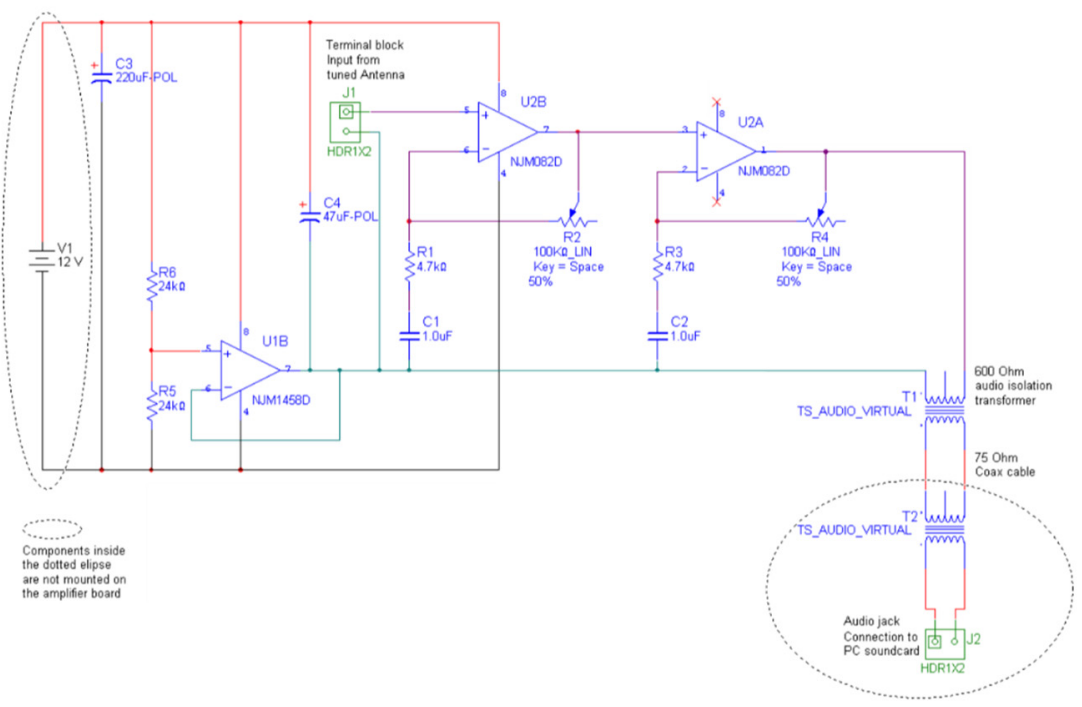

Fig 1: Schematic diagram of amplifier circuit

\subsection{Data Collection}

'Spectrum Lab' software, a free specialised audio analyser package by 'DL4YHF', is used. It digitises the signal received at the sound card from the antenna and performs a fast Fourier transform on it to produces charts of the power against time by reading the log files and graphing them.

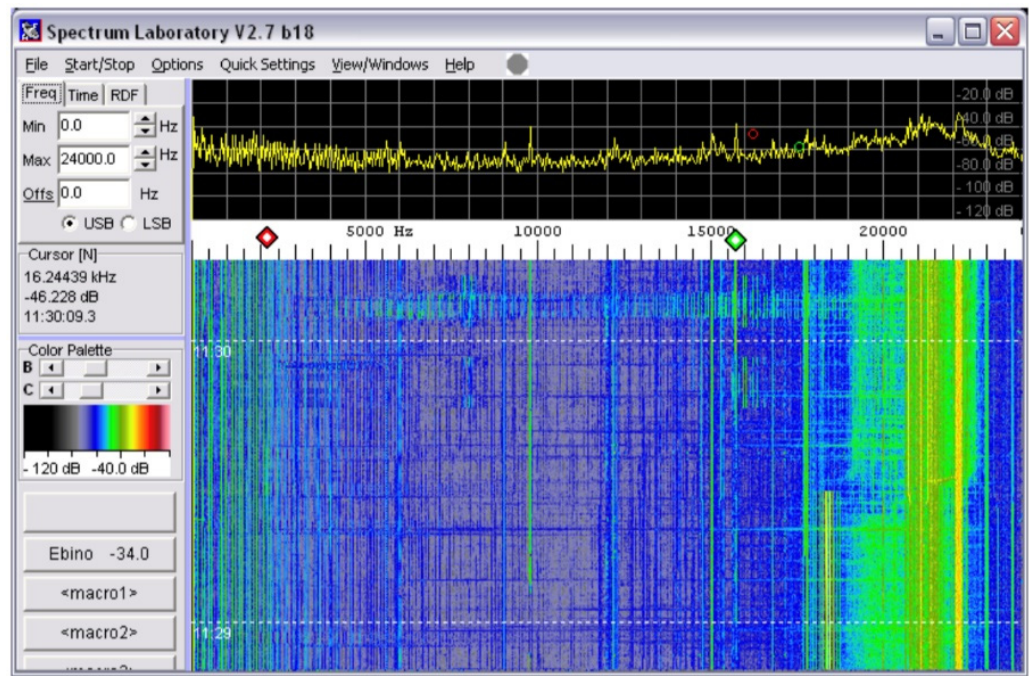

Fig 2: 'Spectrum Lab’ software 


\section{Results}

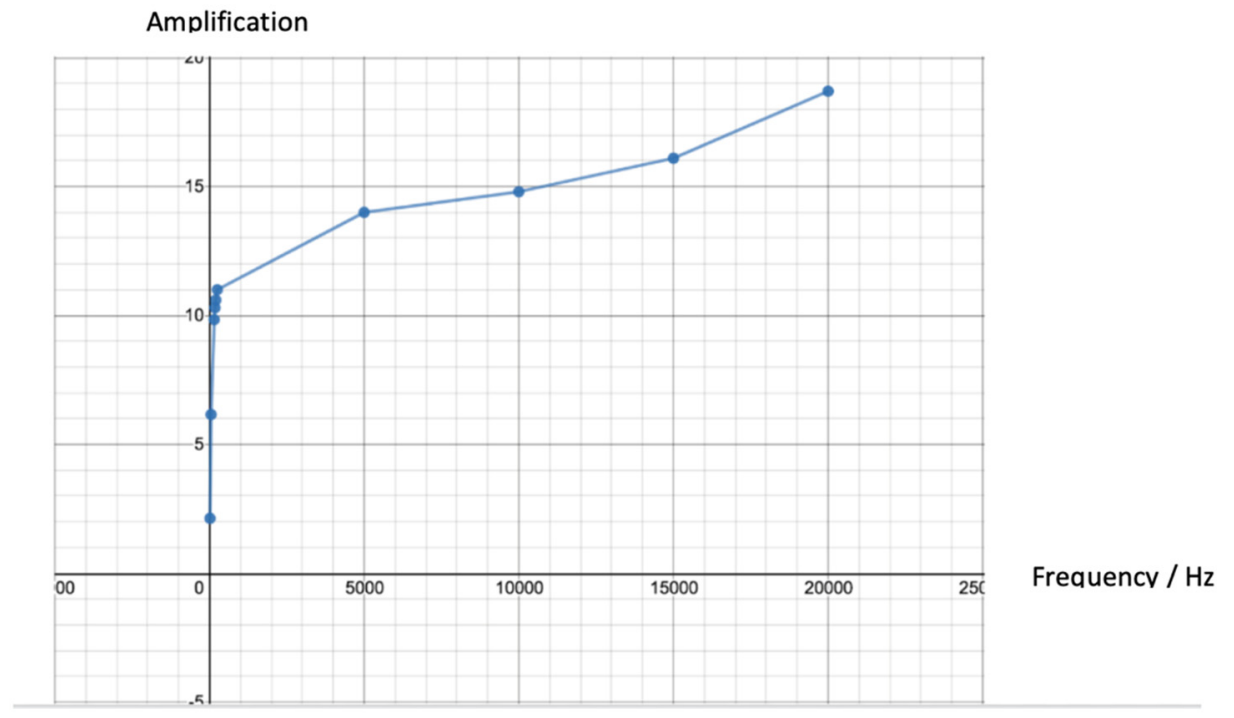

Fig 3: Amplification achieved at various frequencies

\subsection{Conclusion}

From the results, we can conclude that the construction was successful as the signal's peak to peak was amplified across the wide range of frequencies. However, it can also be observed that as the signal frequency increases, so does the amplification. This could be due to the fact that electromagnetic force (EMF) is proportional to signal frequency.

Circuit construction was successful with breadboard as well as amplifying test turning up positive results. The circuit was able to consistently amplify the signal fed to it at multiple frequencies as such it can be concluded that the circuit assembly was successful.

This implies that such a low-cost and effective do-it-yourself alternative to the rather costly radio telescope can be deemed feasible and may even be adopted by radio enthusiasts and amateur astronomers alike not only for VLF observations but possibly further and better modification as well.

With the low-cost, mass production may be an option and could very well imply the possibility of setting up an SSRT array island-wide should others choose to adopt this idea and cooperate with each other on the sharing of data. 


\section{References}

1. Percival Andrews, How to Build Your Own Radio Telescope (2007)

2. Tracking Solar Flares. (n.d.). Retrieved from, $\underline{\text { http://solar- }}$ center.stanford.edu/SID/activities/ionosphere.html

3. SPACEARTH TECHNOLOGY. (n.d.). Ionospheric physics. Retrieved from, http://www.spacearth.net/?page_id=308 